\title{
Das Rektumkarzinom: Fortschritte durch interdisziplinäre Behandlung
}

\author{
Hans-Rudolf Raab ${ }^{a}$ Markus W. Büchler ${ }^{b} \quad$ Rolf Sauer $^{c}$ \\ ${ }^{a}$ Klinik für Allgemein- und Visceralchirurgie, Klinikum Oldenburg, \\ ${ }^{b}$ Allgemeine, Viszerale und Unfallchirurgie, Universität Heidelberg, \\ ${ }^{\mathrm{c}}$ Klinik und Poliklinik für Strahlentherapie, Universität Erlangen-Nürnberg, Erlangen, Deutschland
}

Noch vor zwei Jahrzehnten war die Therapie des Rektumkarzinoms nahezu ausschließlich eine chirurgische. Nur in sehr weit fortgeschrittenen inkurablen Situationen kamen außerhalb von Studien Strahlen- oder Chemotherapie zur Anwendung, jedoch mit nur bescheidenen Erfolgen. Heute ist das Rektumkarzinom ein Paradebeispiel dafür, wie durch interdisziplinäre Maßnahmen in Prophylaxe, Diagnostik und Therapie entscheidende Verbesserungen erreicht werden können. Die verbesserten diagnostischen Verfahren, insbesondere die Videokoloskopie, ermöglichen nicht nur die exakte Diagnose eines kolorektalen Karzinoms sondern auch den Einsatz der Methode im Rahmen der Vorsorge, um Karzinome im Vorund Frühstadium zu erkennen. Für die Vorsorge und für spezielle diagnostische Fragestellungen geht der Weg noch weiter in Richtung auf virtuelle Verfahren, die freilich derzeit noch nicht im Routineeinsatz sind.

Auch die Chirurgie selbst hat in den beiden vergangenen Dekaden entscheidende Fortschritte gemacht. Überkommene Techniken wie das stumpfe Auslösen des Rektums aus der Excavatio sacralis, die zum Teil zu enormen Lokalrezidivraten führten, sind weitgehend verlassen. Die Chirurgie des Rektumkarzinoms ist heute auf einem hohen Niveau standardisiert. Das Hauptschlagwort in diesem Zusammenhang ist die totale mesorektale Exzision. Eine Technik, die, wenn damals auch nicht so benannt, bereits in den 1930er Jahren von Westhues und Götze inauguriert und empfohlen wurde, sich aber erst in den 1990er Jahren durch die Arbeiten von Heald und anderen durchsetzen konnte. Wir haben durch zahlreiche Untersuchungen gelernt, dass der individuelle Chirurg ein wichtiger - wenn nicht der wichtigste - Prognosefaktor in der Therapie des Rektumkarzinoms ist. Dies wird auch wahrscheinlich so bleiben. Die ausgedehnte technische Diskussion der 1990er Jahre, die zum Teil immer noch anhält, hat aber dazu geführt, dass sich das Gesamtniveau verbessert hat. Wie alle Tumoren des linksseitigen Kolons ist auch das Rektum- karzinom einem laparoskopischen Vorgehen zugänglich. Grundsätzlich kann die Operation laparoskopisch genauso ausgeführt werden wie offen. Allerdings kann es Probleme geben, gerade bei den sehr tiefen Resektionen und auch bei großen lokal fortgeschrittenen Tumoren. So bleiben namhafte Chirurgen dem Verfahren gegenüber weitgehend skeptisch und bevorzugen die Tumorpräparation am offenen Situs. Trotz einiger ermutigender Studiendaten kann das Verfahren aus diesen Gründen noch nicht zur allgemeinen Anwendung empfohlen werden.

Die neoadjuvante und adjuvante Therapie des Rektumkarzinoms, Anfang und Mitte der 1990er Jahre noch heiß diskutiert, ist heute weitestgehend unumstritten. Wahrscheinlich kann auch nach optimaler Chirurgie noch ein Zusatznutzen für die Patienten erreicht werden. Unsere eigene deutsche Studie konnte zuletzt zeigen, dass eine neoadjuvante RadioChemotherapie dem postoperativen Vorgehen deutlich überlegen ist. Die Rate an Lokalrezidiven innerhalb der ersten 5 Jahre konnte im Vergleich von post- versus präoperativer Anwendung von 13 auf $6 \%$ mehr als halbiert werden. Gleichzeitig wurden sowohl die akute als auch die chronische Toxizität signifikant vermindert. Darüber hinaus konnte gerade in den kritischen, primär fraglichen Fällen signifikant häufiger der Sphinkter erhalten werden. Insgesamt hat sich der Weg einer konventionell fraktionierten, neoadjuvanten RadioChemotherpaie zunehmend etabliert. Für viele so behandelte Fälle kommt die diskutierte Alternative einer Kurzzeit-Vorbestrahlung mit $5 \times 5$ Gy von vorneherein nicht in Betracht, weil dadurch keine Tumorverkleinerung, also auch keine Erleichterung des operativen Vorgehens und keine Verbesserung des Sphinktererhaltes zu erwarten ist und das Verfahren nicht sinnvoll mit einer Chemotherapie kombiniert werden kann. Für einige Situationen, z.B. Karzinome des mittleren Rektumdrittels, die nicht als T4-Karzinome eingeschätzt werden, bleibt der Stellenwert noch genau zu bestimmen. Die Ergeb-

\begin{tabular}{ll}
\hline KARGER & @ 2005 S. Karger GmbH, Freiburg \\
Fax +4976145207 14 & Accessible online at: \\
$\begin{array}{l}\text { E-mail Information@Karger.de } \\
\text { www.karger.com }\end{array}$ & www.karger.com/cga
\end{tabular}

Prof. Dr. Hans-Rudolf Raab Klinikum Oldenburg gGmbH

Klinik für Allgemein- und Visceralchirurgie

Dr.-Eden-Str. 10, 26133 Oldenburg, Deutschland

Tel. +49 441 403-2254, Fax -2752

E-Mail a-chirurgie@klinikum-oldenburg.de 
nisse laufender Studien müssen abgewartet werden. Weitere offene Fragen, die in Zukunft geklärt werden müssen, sind z.B. die Intensivierung der systemischen Chemotherapie zur Therapie von klinisch okkulten Fernmetastasen und der eventuelle Verzicht auf die Radiatio bei Tumoren des oberen Rektumdrittels.

Beim metastasierten Tumorleiden konnte durch die Ausweitung der Metastasenchirurgie an Leber und Lunge doch noch für einen substantiellen Anteil der Patienten eine positive Langzeitprognose, in nicht wenigen Fällen sogar eine Heilung erreicht werden. Auch hier etablieren sich zunehmend interdisziplinäre Konzepte, z.B. mit einer neoadjuvanten Chemotherapie der Lebermetastasen. Es hat sich gezeigt, dass ursprünglich technisch irresektable Metastasen durch eine Vorbehandlung mit Oxaliplatin-, gegebenenfalls auch Irinotecanhaltigen Schemata deutlich verkleinert werden können. Auch wenn Vollremissionen selten sind, kann auf diese Weise doch in einigen Fällen noch Resektabiltät erreicht werden. In dieser Beziehung noch ganz am Beginn steht die Entwicklung von Radiochirurgie und Stereotaktischer Radiotherapie von Lun- gen- und Lebermetastasen. Die genannten neuen Chemotherapeutika, Oxaliplatin und Irinotecan, sowie auch Antikörper gegen den EGF-Rezeptor, wie Cetuximab, oder der antiangio-genetische Antikörper Bevacizumab scheinen auch wesentlich zu einer Verbesserung der Erfolge bei den nur palliativ therapierbaren Patienten beizutragen.

Ein großer Teil der hier nur skizzierten immensen Fortschritte in der Therapie des Rektumkarzinoms in den vergangenen zwei Jahrzehnten konnte allein durch interdisziplinäre Bemühungen erreicht werden. Vieles davon fand seinen Niederschlag im Rahmen einer S3-Leitlinie zur Therapie des kolorektalen Karzinoms, die im Auftrag der Deutschen Krebsgesellschaft und im Konsens mit allen beteiligten Disziplinen im vergangenen Jahr erarbeitet wurde.

Daher begrüßen wir es außerordentlich, dass die Zeitschrift Chirurgische Gastroenterologie InTERdiziplinär sich zu einem Themenheft zum Rektumkarzinom entschlossen hat. Als Gastherausgeber haben wir uns bemüht, der großen Breite des Themas gerecht zu werden und insbesondere die interdisziplinären Aspekte zu betonen. 